\title{
SEAMHex - Fast 6D Additive Manufacturing using an innovative Screw Extruder applied on a Hexapod Parallel Kinematic
}

\author{
Steffen Ihlenfeldt ${ }^{1), 3)}$, Welf-Guntram Drosse ${ }^{2), 3)}$, Martin Kausch ${ }^{3)}$, Christian Friedrich ${ }^{1), 3)}$, Torben \\ Wiese $^{3)}$, Lysander Jankowsky ${ }^{3)}$ \\ 1) TU Dresden, Institute for Mechatronic Engineering, Helmholtzstrasse 7a, 01167 Dresden; \\ Steffen.Ihlenfeldt@tu-dresden.de, christian.friedrich@tu-dresden.de, werkzeugmaschinen@tu- \\ dresden.de \\ 2) Chemnitz University of Technology, Institute for Machine Tools and Production Processes, \\ Reichenhainer Strasse 70, 09126 Chemnitz \\ 3) Fraunhofer Institute for Machine Tools and Forming Technology, Reichenhainer Strasse 88, \\ 09126 Chemnitz; info@iwu.fraunhofer.de, lysander.jankowsky@iwu.fraunhofer.de, \\ torben.wiese@iwu.fraumhofer.de
}

\section{Keywords}

Additive Manufacturing, 3D Printing, Machine Tools, Parallel Kinematics, Screw Extrusion

\begin{abstract}
The Screw Extrusion Additive Manufacturing (SEAM) technology provides output rates up to $10 \mathrm{~kg} / \mathrm{h}$, a melt pressure up to 350 bar and temperatures up to $400{ }^{\circ} \mathrm{C}$ as well as strands with an adjustable bead width between 1-8 $\mathrm{mm}$. Due to a bypass nozzle, position jumps without material extrusion, local wall thickness reduction, and the control of the volume flow (0-100\%) are made possible. Further, the extruder is able to process fiber-reinforced as well as highly filled plastics and integrates a regranulation system to return bypassed material into the process. The hexapod parallel kinematic meets the process requirements, as it generates a rapid movement of the workpiece (up to $1 \mathrm{~m} / \mathrm{s}$ ) in up to 6 degrees of freedom within a large printing workspace $\left(1100 \times 800 \times 600 \mathrm{~mm}^{3}\right)$, where an additional Z-axis carries the extruder and realizes the part height. Eccentric joints provide high accuracy and stiffness while being cost-efficient at the same time. The commercial Beckhoff TwinCAT control allows G-code processing and provides an HTML based human machine interface (HMI) for machine and extruder control. Consequently, high accuracy is achieved, which is verified by the use of a double-ball-bar measuring device and by producing a test workpiece.
\end{abstract}

\section{Introduction and Approach of the SEAM technology}

The field of 3D printing of thermoplastic components is currently partitioned in a large number of processes, such as powder bed fusion (PBF), material extrusion (MEX), material jetting (MJT) or vatphotopolymerization (VPP) [1-3]. In material extrusion, the fused filament fabrication process (FFF), in particular, impresses with its simple and inexpensive process principle and the ability to process selected standard materials. In 1989 Scott Crump [1] submitted the patent for the 3D printing process via Fused Deposition Modelling (FDM) [4] and is therefore considered to be co-founder of today's FDM 3D printing. In this process, the thermoplastic filament is plasticized through a melt nozzle (hot end) via a feed unit and applied in layers to the building platform. Higher processing speeds are limited by the low plasticizing performance of the hot ends and a relatively slow positioning system of the printing head.

Today, the production of complex large plastic components in small series is very laborious and expensive. Established 3D printing processes for the production of plastic components are unprofitable for large series, as process times of up to several days and material prices of more than $40 \mathrm{EUR} / \mathrm{kg}$ lead

Digital Object Identifier: http://dx.doi.org/10.21935/tls.v5i1.166

www.lightweight-structures.de

This is an open access article under the CC BY 4.0 license (http://creativecommons.org/licenses/BY/4.0/) 
to high costs $[5,6]$. For this reason, extrusion-based 3D printing has been developed with the help of an extruder screw and is already known from various systems and publications. Manufacturers of such systems are, for example, CEAD, Titan Robotics LTD, Thermwood, or BAAM Cincinnati. The material is plasticized by an extruder screw and extruded by a movable nozzle. These processes achieve output rates of up to $250 \mathrm{~kg} / \mathrm{h}$ and extrude very thick strands with a cross section of up to $25 \times 5 \mathrm{~mm}$. Component dimensions of $6 \mathrm{~m}$ in length, $2.4 \mathrm{~m}$ in width and $1.8 \mathrm{~m}$ in height can be produced and, thus, belong to the largest printing systems that currently exist [6]. To achieve high productivity with high strand diameters, appropriate deposition speeds and a cooling system is required. Due to the thick crosssection strands, these processes allow limited dimensional accuracy, especially in corners when there is no volume flow reduction, and a very rough component structure that is often smoothed by a subsequent milling process.

This compromise between accuracy and speed is the field where the Screw Extrusion Additive Manufacturing (SEAM) technology operates. SEAM stands for Screw Extrusion Additive Manufacturing and its technology, with an output rate of up to $10 \mathrm{~kg} / \mathrm{h}$, is characterized by the fact that relatively thin strands, e.g. to process with a cross section of $3 \times 0.8 \mathrm{~mm}$, in combination with a very fast movement system with speeds of up to $1 \mathrm{~m} / \mathrm{s}$ can be realized. This enables optimum productivity and attention to detail as well as surface quality to be achieved through rapid solidification of the melt strands. In addition, by plasticizing the thermoplastics with an extruder screw, a melt pressure of up to 350 bar is achieved and through a nozzle with a variable diameter, a strand width of $1-8 \mathrm{~mm}$ can be printed. With such a high system pressure, fiber-reinforced and highly filled plastics can also be processed and process pressures of up to 50 bar can be generated between the nozzle and the coating layer. In this way, very high layer adhesion between the strands but also, when printing onto inserted semi-finished products (textiles, sheets and foils of different materials), a high material density with a low proportion of pores are achieved. In addition, when compared to other extrusion-based 3D printing processes, the SEAM technology is characterized by the fact that the volume flow of the melt can be controlled between 0 and $100 \%$. A bypass nozzle system enables positional jumps without material extrusion and wall thickness reductions. The output rate can also be adapted to the printing speed, e.g. when reducing speed at corners or small part geometries [5].

\section{Technology Demonstrator SEAMHex}

Plasticizing unit:

Mass flow up to $7 \mathrm{~kg} / \mathrm{h}$

- Volume flow control

Machine:

- Compact and portable $2.2 \times 3.0 \times 2.8 \mathrm{~m}^{3}$

- Only power supply needed

- Melt temperatures up to $400^{\circ} \mathrm{C}$

- Bead width $1 \ldots 8 \mathrm{~mm}$

- Exchangeable screws

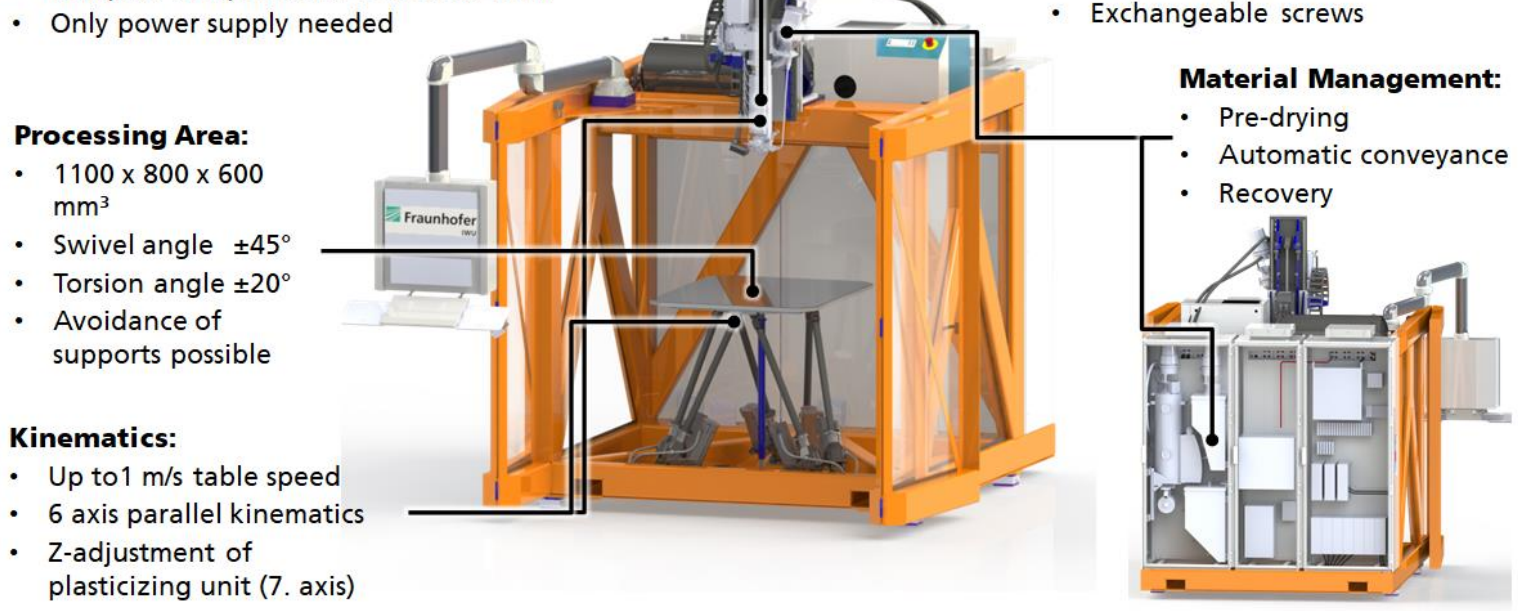

\section{Figure 1: SEAMHex technology demonstrator with machine specifications}

As new contribution, this paper presents the automated, fully integrated additive manufacturing technology demonstrator SEAMHex that brings the SEAM technology into application, Figure 1. The allin-one demonstrator, which includes plasticizing unit, moving device, control, and material handling, provides a fully controllable volume flow, a double valve system with bypass flow and a regranulation system combined with a hexapod machine tool that allows accurate movement in 6 degrees of freedom. Hereby, for the first time eccentric joints are used within a hexapod-based printer to ensure high stiffness and accuracy. In addition, the accuracy assessment by a double-ball-bar with extended range bases on a new measuring device and procedure. 
After a short requirement overview (Section 2), this paper presents the design of Plasticizing Unit, Hexapod Machine Tool, and Material Handling (Section 3). Subsequently, the control system including hexapod and extruder control as well as path planning via a CAM system are discussed (Section 4). Finally, direct and indirect accuracy assessments using a double-ball-bar measuring system and a test workpiece, respectively, are presented (Section 5).

\section{Process and Machine Requirements}

During the development of the SEAMHex, various process capability requirements were challenging. On the one hand, the high-performance SEAM technology requires a movement speed of up to $1 \mathrm{~m} / \mathrm{s}$ in order to produce small component contours with the high mass output. On the other hand, the printing space must be large enough to produce components with dimensions up to $1100 \times 800 \times 600 \mathrm{~mm}^{3}$. Further, the necessary velocities also require high accelerations with regard to a dynamic movement and many direction changes during the printing process. The possible dynamic in radii and spline curves while printing depend on the dynamic of the machine. Thereby, imprecise movements directly affect the printing quality. As during direction changes a velocity reduction occur, the bypass system need to compensate the material output to uphold the strand width. In addition, the SEAMHex should also be able to use the advantages of multiaxial 3D printing; therefore, 5 degrees of freedom (dof) are necessary in the movement. In this case, support structures can be reduced or even avoided and it is possible to print on a curved plane or insert components. Furthermore, it enables ideal and continuous trajectories with a reduction of start-stop situations as well as a reduction of the step effect in the case of diagonally arranged structures [7].

Especially in the field of large-scale additive manufacturing, accuracy becomes more difficult to manage, because usually the printed strands have a large diameter. The surface roughness increases with the strand height, because every layer is visible. Smaller strand diameters decrease the surface roughness, but slow down the whole process. A solution is a small layerheight and high velocities at the same time and further, if necessary, a great strand width. The heat management becomes more and more important, when the printed strands or the printed specimens become bigger. High temperatures in the polymer strands result in inner tensions and warpage and affect the dimensional accuracy. To reduce warpage and tension, cooling systems, or higher layer cycle times are necessary. Fibre filled polymers can also reduce warpage, because they stabilize the printed specimen [8]. For a high accuracy in the printing process, it is important to have an adequate calibration between the plasticizing unit and the building platform, because a hundredth of a millimeter change in the layer thickness leads to a change in width of a tenth of a millimeter, which leads to poor accuracy.

\section{SEAMHex Machine Design}

In cooperation with the Institute for Mechatronic Engineering of the TU Dresden, the Fraunhofer Institute for Machine Tools and Forming Technologies has developed and built the SEAMHex Figure 2. To match process and machine requirements, a hexapod has been selected as motion unit. It allows a movement in six degrees of freedom and provides a large workspace. Since the SEAM plasticizing unit is significantly heavier than the plastic components to be printed, the workpiece is moved by the hexapod to improve dynamics. To take full advantage of the hexapod's movability, the extruder is mounted on an additional linear axis. This enables the printing of significantly higher components. 


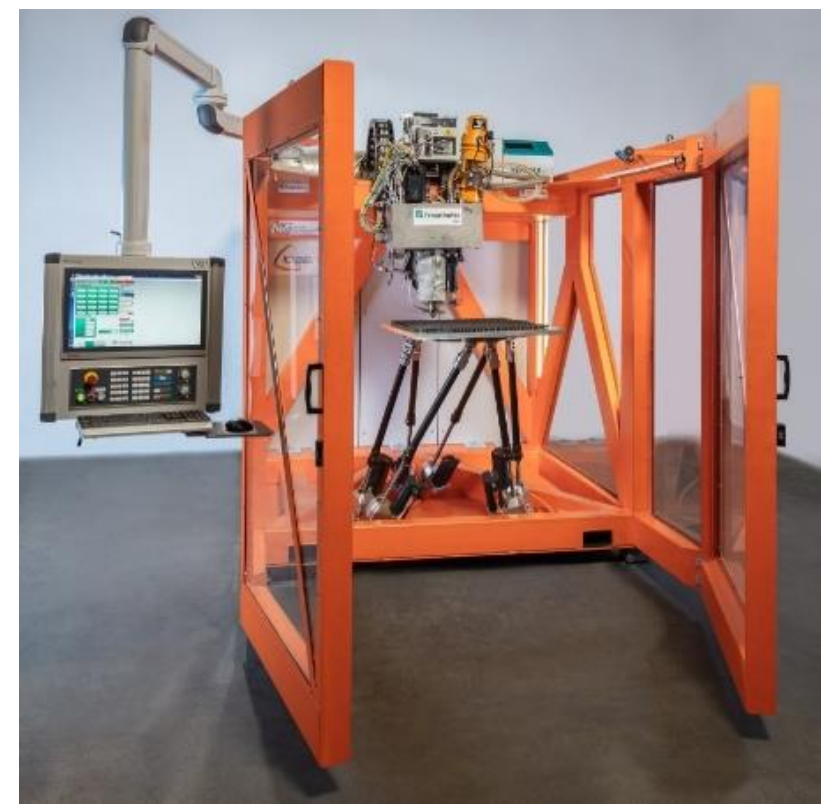

Figure 2: Buildup SEAMHex demonstrator

\subsection{Plasticizing Unit}

The plasticizing unit, Figure 3 left, consists of a special extruder screw, a temperature-controlled screw cylinder, a bypass, and a main nozzle, as well as a material feed. The screw cylinder is heated by heating bands in three temperature zones and, if necessary, cooled by compressed air. The temperature setup is done by the operator at the machine control and is set to the respective material. The machine control regulates the heating devices to the set temperature at every moment. In order to ensure constant material output, the extruder has a main nozzle and a bypass nozzle. There is a rotary piston in the bypass assembly, which regulates the mass flow from the main nozzle between 0 and $100 \%$. With a reduced mass flow from the main nozzle, the excess material is extruded radially through the bypass nozzle. Consequently, the pressure at the main nozzle remains nearly constant with a variance of \pm 1 bar. As pressure fluctuations have a direct effect on the strand width, the bypass nozzle increases the precision of the printing process significantly. The piston is controlled by the program code of the path control. The melt pressure is measured, monitored, and displayed before the piston. The limiting values for the melt pressure are permanently programmed. As a safety system, the machine is forced to shut down, if the melt pressure exceed the limiting values. Another safety system is a bursting pin that is placed opposite the pressure sensor, which is triggered at a melt pressure of more than 500 bar, and allows the melt to flow out in a controlled manner. The main nozzle can be adapted to the construction job by using various screw nozzles. These screw nozzles differ in their opening diameter, as well as the screw nozzle plate, which enables strand widths of up to $8 \mathrm{~mm}$. 

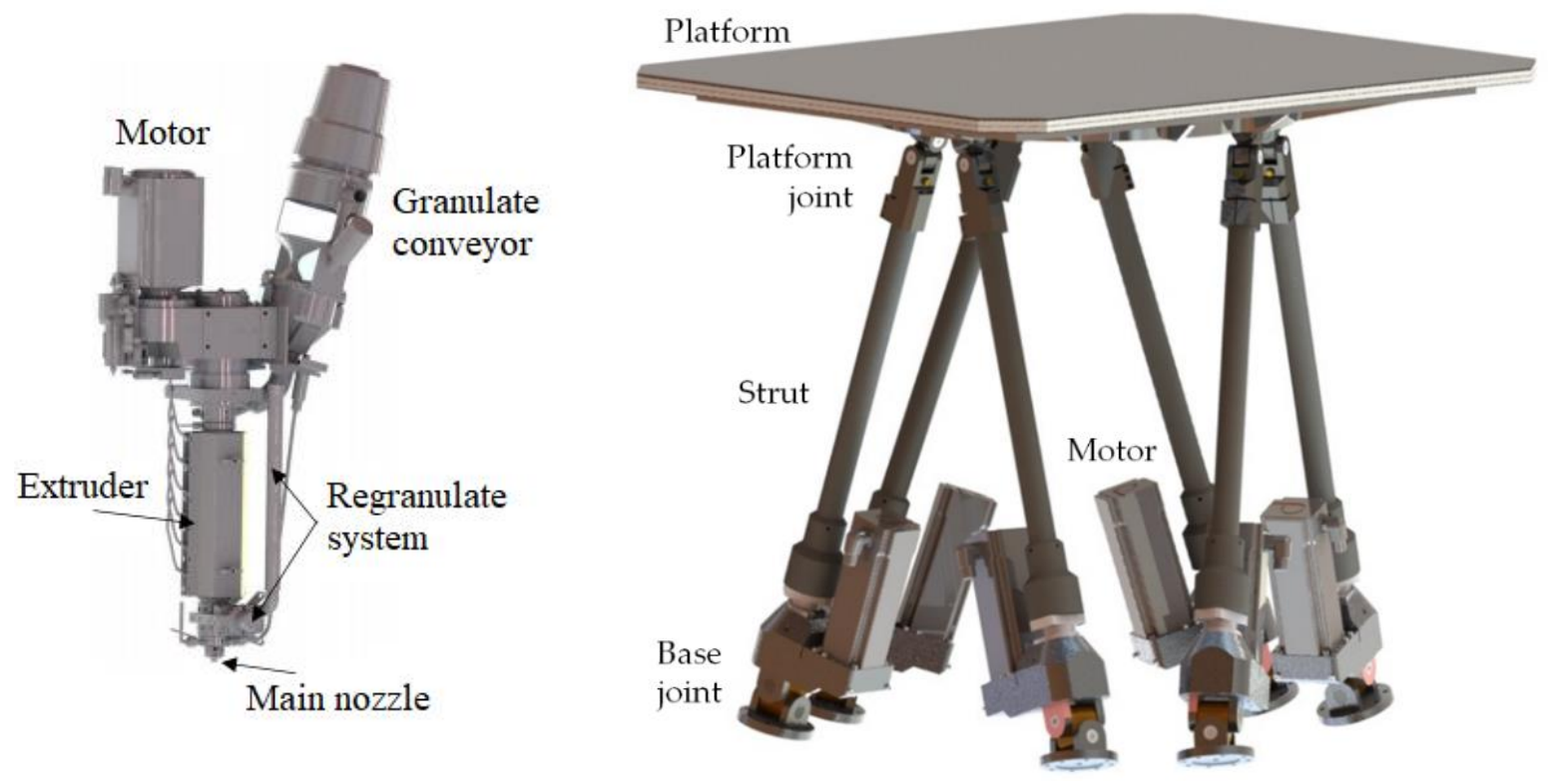

Figure 3: CAD of the Plasticizing Unit including material feed and partial regranulate system (left) and the Hexapod including the printing bed (right)

Due to the extensive periphery of the extruder like conveyor pipelines and electric wires and a tendency to scale the system for higher output rates, the plasticizing unit is heavy. In the current structure, the plasticizing unit weights approx. $100 \mathrm{~kg}$. This results in an inertia of the unit, which is disadvantageous for the high-required speeds, if the plasticizing unit has to move in all directions. For high dynamics, it is not possible to reduce much weight on the plasticizing unit, wires and pipelines and to move the periphery, so a highly dynamic building platform is needed. Another advantage is a relatively fixed working point at the extruder nozzle, because it helps evaluating specific parameters at the same position like temperature, optical measurements, simple constructions for sensors etc.

\subsection{Hexapod Machine Tool}

With respect to the process requirements mentioned above, the positioning device is requested to move the workpiece in at least 5 degrees of freedom, within a large workspace, and, at the same time, provide a compact and lightweight machine design. Further, high positioning accuracy (at least $\pm 100 \mu \mathrm{m}$ ), high velocity $(1 \mathrm{~m} / \mathrm{s})$, and adequate dynamics including small moving masses are required, whereas the demands regarding process forces are rather small.

These requirements are met best by an optimized version of a hexapod parallel kinematic, as presented in the following. The hexapod consists of six struts of variable length that are mounted between a fixed base frame and the moving end-effector platform, Figure 3 right. Each strut contains of servomotor, belt drive, and spindle drive that represent the active prismatic joint, as well as five passive rotational joints, Figure 4. For the presented application, the 3D printing process takes place at the top of the hexapod end-effector, which carries the workpiece, while the plasticizing unit is moved by an additional Z-axis that is fixed at the base, Figure 1 and Figure 2.

Hexapod parallel kinematics have been topic of many works and have many benefits, such as 6-dof movement, light-weight machine design and moderate costs, where other characteristics, for example accuracy and stiffness as well as the complex workspace, can be challenging $[9,10]$. In particular, the design of the joints influence these parameters, which led to complex and expensive universal joint designs [11]. The approach of a hexapod of simple design [12-14], on the other hand, prefers cheap components and allows systematic errors, which are compensated for by geometric, elastostatic, and thermal error models. Nevertheless, for some influences, such as non-linear joint play, the approach is limited. Consequently, new eccentric joints have been developed $[15,16]$ that provide higher positioning accuracy and much higher stiffness, when compared to conventional joints [17, 18]. At the same time, they are cost-efficient and, therefore, meet the approach of simple design. In case of eccentric joints, the rotational axes do not intersect anymore, which provides larger construction volume for cardan cross and bearings, and, in the consequence, allow the optimized design. On the contrary, the approach leads 
to a more complex mathematical solution of the forward and backward kinematic problem as well as to lateral forces and moments upon the struts, which is why eccentric designs have been avoided for a long time [16]. Figure 4 shows the build of a single hexapod strut including the spindle drive and eccentric joints. Further details regarding the approach and mathematics are presented in [16]; details to accuracy and stiffness in $[17,18]$.

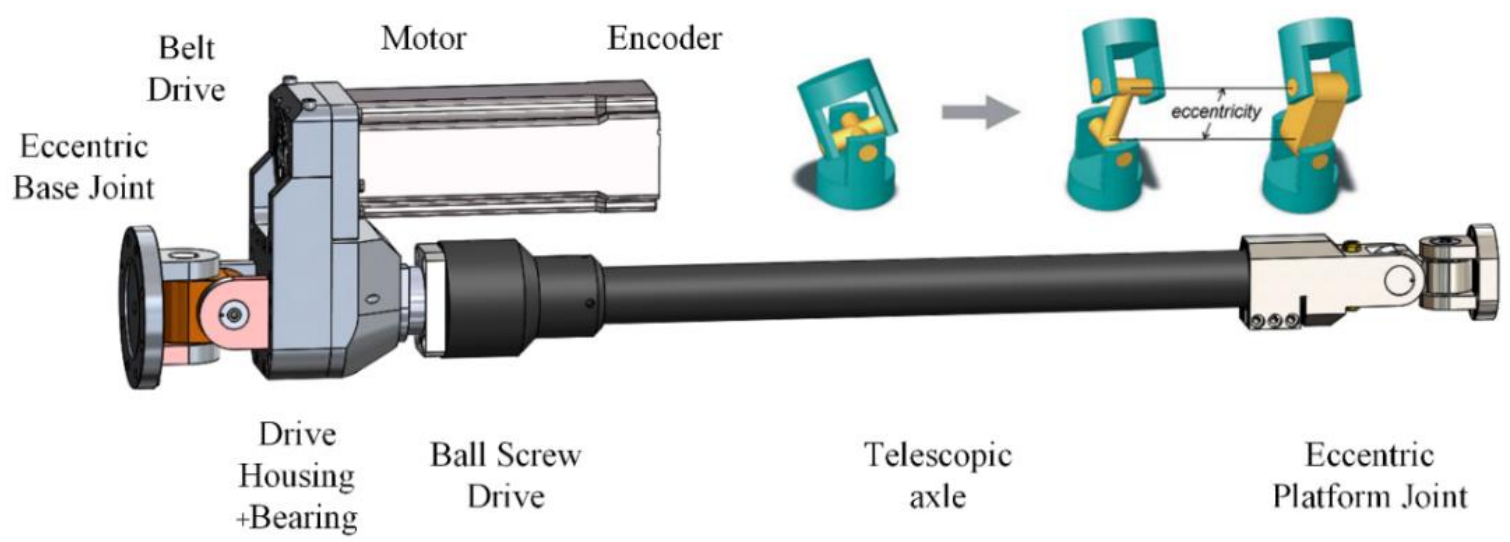

Figure 4: Single hexapod strut with eccentric joints (Length: 875-1470 mm, Motor: $5.65 \mathrm{Nm}$, Spindle pitch: $20 \mathrm{~mm}$, Eccentricity: $32 \mathrm{~mm}$ and $38 \mathrm{~mm}$ at base and platform joint, resp.)

Another characteristic of parallel kinematics regarded within the demonstrator design is the complex and orientation-dependent workspace that is determined by strut length limits and joint limits. Figure 5 presents the hexapod positioning workspace without inclination and with an inclination of $25^{\circ}$. As the height of the hexapod workspace (Z-direction, blue) is rather limited, the hexapod operates mainly in 5 dof at optimal Z-position and an additional Z-axis is used to realise the workpiece height. Beside the machine design, the workspace simulation can also be used for optimal part placement and path generation.
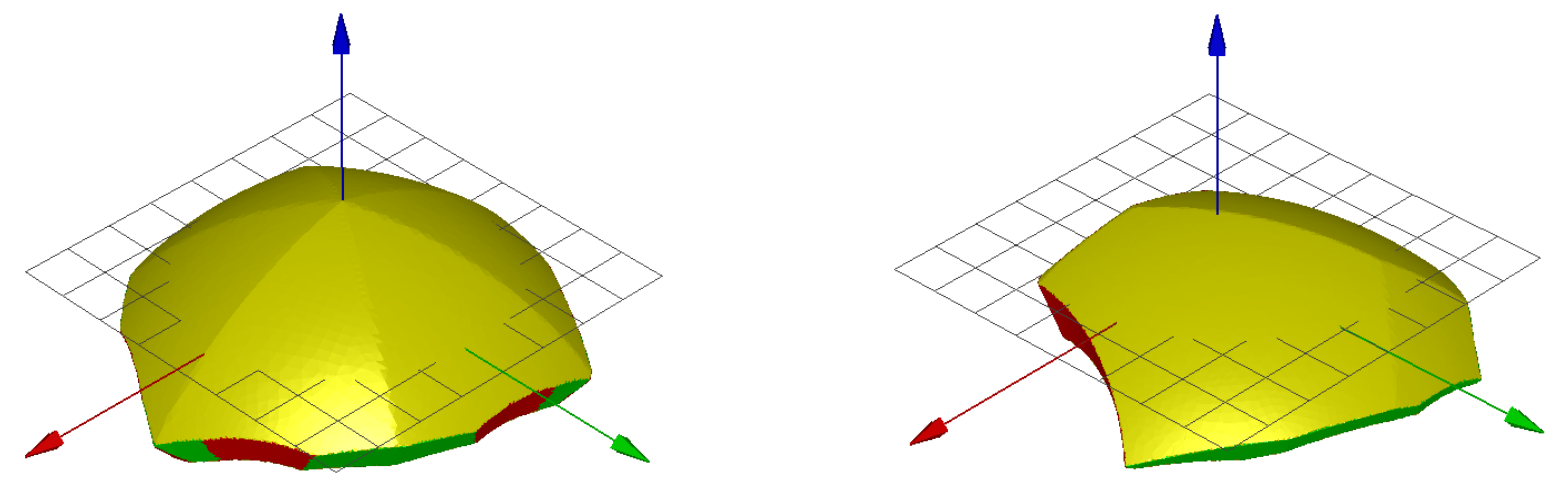

Figure 5: Positioning workspace of the hexapod at zero orientation (left) and at $25^{\circ}$ inclination around $X$ (right) without including the additional Z-Axis; Grid size: $200 \mathrm{~mm}$; Coordinate axis: $X$ (red), Y (green), Z (blue); Limits: strut lengths (yellow), base joints (green), platform joints (red)

Finally, the hexapod design allows optional force sensor integration for future applications [19, 20]. In conclusion, the SEAMHex machine tool provides 6 dof as well as velocity and acceleration rates that are suitable for the novel printing head. Further, high stiffness and accuracy are achieved through eccentric joints, a large workspace through additional z-axis, and, finally, a good usability through ability of G-code based control, as presented in the next section.

\subsection{Machine Frame and Material Handling}

Since the SEAMHex has been planned as a mobile demonstrator, it has a compact machine frame that includes all components necessary for operation. This allows a fast and flexible transport by crane or forklift. Material storage, dryer, and compressor for air supply are fully integrated. The SEAMHex only needs a 400 VAC, 63A power supply. 
To supply the extruder with raw material, an automated conveying system is integrated, which is shown schematically in Figure 6. The material is transported from the storage to the extruder via granulate conveyors. Prior to this, drying takes place to reduce the moisture content of the material. Further, a regranulate system is integrated, which can mix the excess material from the extruder bypass with new raw material.

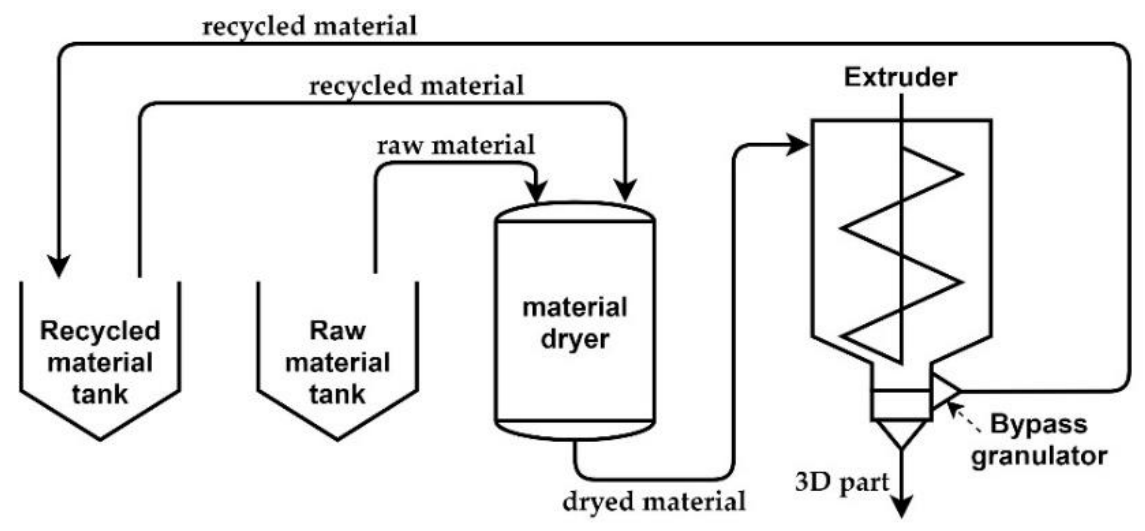

Figure 6: Scheme of material handling

For optimum use of space, the power and control electronics as well as the dryer and the storage tanks for the raw and regranulate material are housed in a series of cabinets. The printing chamber can be viewed from three sides through glass panels and the operating panel can be guided around the entire machine via a support arm. This allows the operator to easily observe and control the running process.

\section{Control System}

In production and for extrusion-based 3D printing, the use of computerized numeric control (CNC) has proven itself as highly reliable. Here, it enables the synchronous automated path calculation for all 7 positioning axes. Consequently, exact path movement and high printing quality is achieved. Furthermore, a CNC control is able to process standardized program code (G-code) for the control of the movement and the process. As a result, a broader understanding among machine operators is achieved and CAM programs required for slicing only need to be adapted to the machine system to a minor extent. An adapted CAM program is required to automate the programming of the G-code for the printing of parts, thus significantly accelerating the production cycle. For the process control of the SEAM plasticizing unit, a control must be available, which monitors the different process parameters by sensors and can regulate them by different actuators.

\subsection{SEAMHex and Extruder Control}

As control system, the commercial Beckhoff TwinCAT 3.1 including CNC and programmable logic controller (PLC) are used. The CNC operates the motion system, consisting of the hexapod and the redundant Z-axis. One challenge is the mathematical conversion of the Cartesian movement specifications from the NC program (Cartesian coordinates) into the corresponding strut lengths (joint coordinates). A real-time kinematic transformation of the hexapod with eccentric joints has been programmed in $\mathrm{C}_{++}$for this purpose. In addition, the redundant $Z$-axis must be taken into account in order to use the working area optimal. An algorithm has been programmed in the CNC for this purpose. This allows the path planning in the CNC to take place in the Cartesian space, which is advantageous as an exact path planning including dynamic smoothing is possible and no offline transformation is necessary.

The PLC controls the SEAM process unit that consists of several separately controlled heating zones, an extrusion screw driven by a spindle motor and a controllable double valve system that includes the main printing nozzle and the bypass nozzle. To ensure a homogeneous volume flow during printing, the internal material pressure in the extruder must remain constant. In dynamic printing processes, controlling the screw speed is not sufficient for this purpose. Instead, the pressure is also controlled via the bypass valve. If the internal pressure of the extruder becomes too high, the valve is opened, allowing excess plasticized material to be discharged via a recycling system. Since the extruder pressure is 
directly dependent on the movement of the main nozzle, the bypass nozzle follows the speed of the main nozzle to enable faster control behavior. This results in the control loop shown in Figure 7.

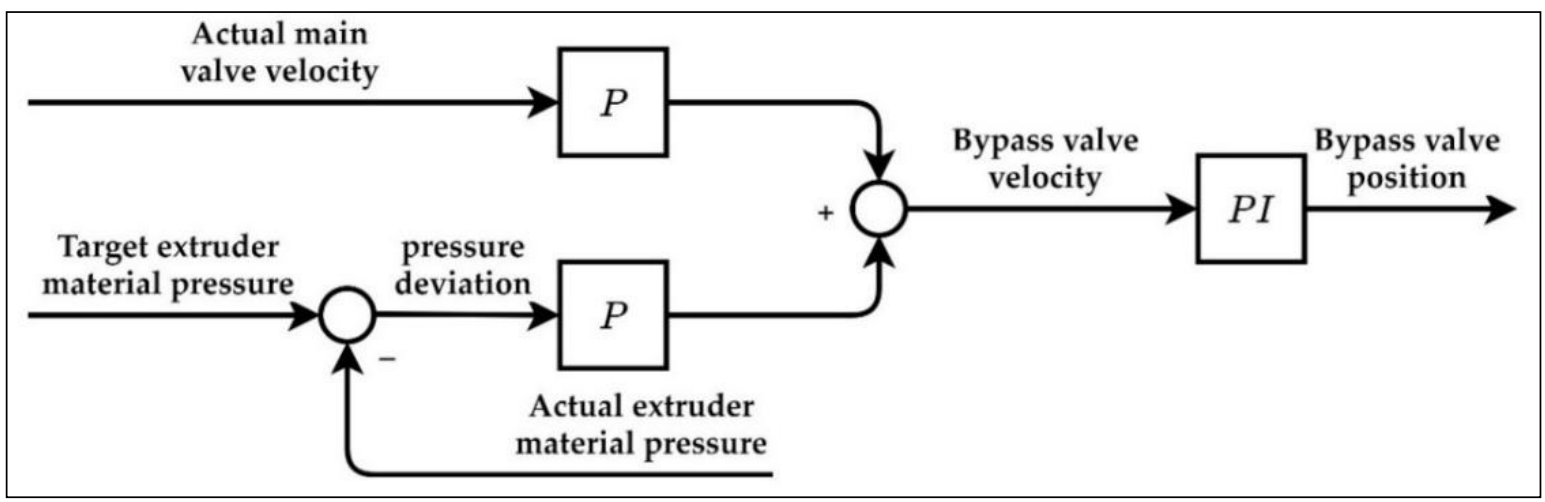

Figure 7: Control loop structure of the bypass nozzle to regulate the material pressure inside the extruder.

To keep the bead width constant when printing small radii in the range of a few millimeters or sharp corners, the volume flow is controlled as a function of the feed speed as can be seen in Figure 8 . For this purpose, the opening of the main nozzle is controlled proportionally to the ratio of actual and target feed rate, as shown in Figure 9.

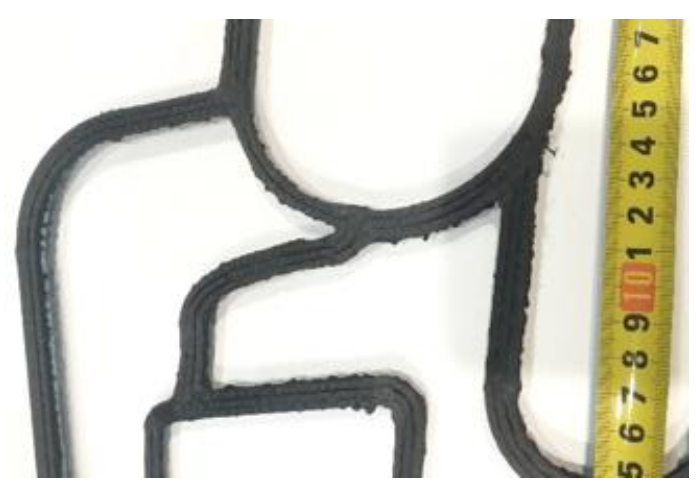

Figure 8: Corners and small radii in a printed specimen, constant strand width in corners, two and three strands printed next to each other.

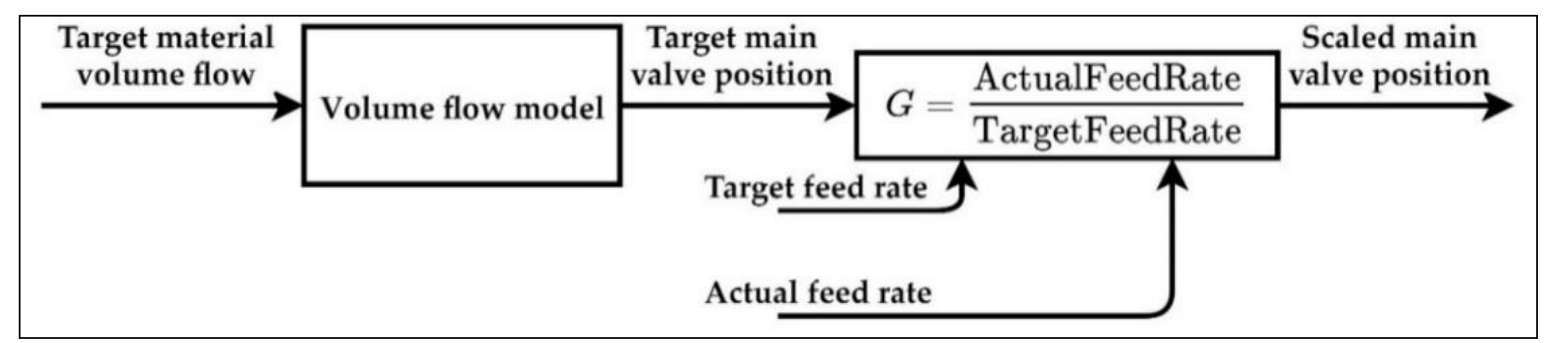

Figure 9: Control structure of the main nozzle to regulate the material volume flow.

For the operation of the SEAMHex, a HTML based human machine interface (HMI) has been programmed. This allows the operation of the CNC in the operating modes automatic, manual and jog mode, the control of the extruder unit temperatures, and the operation of the different conveyors and additional units. Manual process monitoring is possible via various displays. Another advantage of the HTML-based HMI is the possibility to display it on any mobile device via an internet browser. This is especially advantageous during commissioning of the machine or new printing programs. 


\begin{tabular}{|c|c|c|c|c|c|c|c|}
\hline \multirow{2}{*}{ 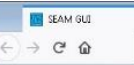 } & \multicolumn{3}{|l|}{$\bar{x}+$} & \multicolumn{4}{|r|}{ ज्ञात्र } \\
\hline & \multicolumn{3}{|c|}{ 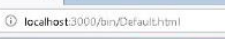 } & \multicolumn{3}{|c|}{$\cdots \oplus$ म $Q_{\text {Sucher }}$} & In $₫ 0 \equiv$ \\
\hline \multirow{2}{*}{\multicolumn{2}{|c|}{$\begin{array}{l}\text { SEAM CONTROL } \\
\text { Ist-/Sollposition: }\end{array}$}} & \multicolumn{2}{|c|}{ EStop } & $\mathrm{CNC}$ & \multicolumn{2}{|c|}{ Extruder } & Aggregate \\
\hline & & \multicolumn{2}{|c|}{ PCS $v$} & \multicolumn{2}{|c|}{ Achsgeschw: } & \multicolumn{2}{|c|}{ Modus | Status } \\
\hline \multicolumn{2}{|c|}{$\mathrm{X}$ Achse 0.000} & & $\mathrm{~mm}$ & 0.0 & $\mathrm{~mm} / \mathrm{s}$ & \multicolumn{2}{|l|}{ CNC: } \\
\hline Y Achse 0.000 & \multicolumn{2}{|c|}{0.000} & $\mathrm{~mm}$ & 0.0 & $\mathrm{~mm} / \mathrm{s}$ & \multicolumn{2}{|c|}{ StandbyMode } \\
\hline Z Achse 0.000 & \multicolumn{2}{|c|}{0.000} & $\mathrm{~mm}$ & 0.0 & $\mathrm{~mm} / \mathrm{s}$ & \multicolumn{2}{|c|}{ Selected } \\
\hline A Achse 0.000 & \multicolumn{2}{|l|}{0.000} & $\operatorname{deg}$ & 0.0 & $\mathrm{deg} / \mathrm{s}$ & \multicolumn{2}{|c|}{ FehleriD: OK } \\
\hline B Achse 0.000 & \multicolumn{2}{|l|}{0.000} & $\operatorname{deg}$ & 0.0 & $\mathrm{deg} / \mathrm{s}$ & \multicolumn{2}{|c|}{ Aggregate errors: } \\
\hline C Achse 0.000 & \multicolumn{2}{|l|}{0.000} & $\operatorname{deg}$ & 0.0 & $\mathrm{deg} / \mathrm{s}$ & & \\
\hline \multicolumn{3}{|c|}{ ZE Achse 0.000} & $\mathrm{~mm}$ & 0.0 & $\mathrm{~mm} / \mathrm{s}$ & & \\
\hline \multicolumn{2}{|c|}{ Extruderdrehzahl } & Ovr. & & senstellur & Ingen: & & \\
\hline \begin{tabular}{l|l}
0.0 & 0.0 \\
\end{tabular} & $\mathrm{rpm}$ & 100 & $\%$ Hau & upt 0.00 & & & \\
\hline Vorschub: & & & & pass 0.00 & 000 & Türen & aeschlossen \\
\hline \begin{tabular}{|l|l|}
0.000 & 0.000 \\
\end{tabular} & $\mathrm{~mm} / \mathrm{min}$ & 100 & $\%$ & & & MBF ge & espert: off \\
\hline NC Handsatz: & & & & & & Extrt & ıderdruck: \\
\hline Hier NC-Cod & schreiben, & am M & MCP bes & estätigen & & 0.0 & 0.0 bar \\
\hline & & & & & & Dise & endruck: \\
\hline & & & & & & 0.0 & bar \\
\hline CNC & $\begin{array}{l}\text { Extruder } \\
\text { Steuerung }\end{array}$ & $\begin{array}{r}\text { Zus } \\
\text { aggr }\end{array}$ & $\begin{array}{l}\text { satz- } \\
\text { regate }\end{array}$ & Deutsc & $\operatorname{ch} \vee \#$ & Fra & $\begin{array}{r}\text { unhofer } \\
\text { IWU }\end{array}$ \\
\hline
\end{tabular}

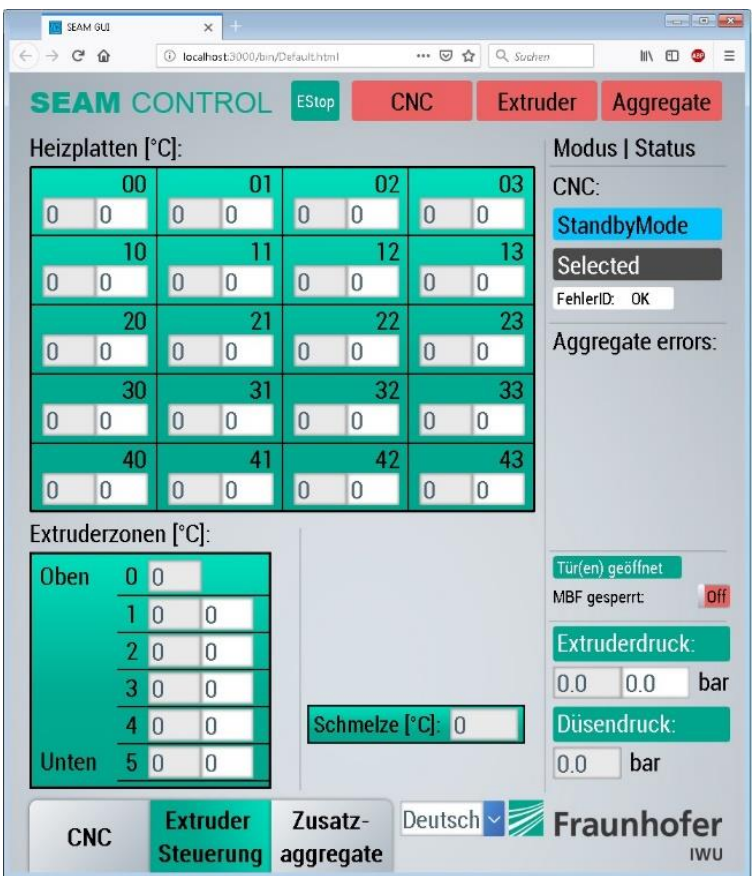

Figure 10: HTML based HMI of the SEAMHex. Left: CNC control; Right extruder and temperature control.

\subsection{DCAM Path Planning Software}

To avoid the enormous effort of manually programming an NC program and to allow more complex part structures to be printed, CAM software systems, so-called slicers, are used for 3D printers. These are able to divide the digital 3D model of a part into several printing layers and generate the corresponding movement path for each layer. In addition, many other options such as printing speed, wall strengths, and filling contours can be set.

For the SEAMHex the DCAM software from SKM is used, which was extended by the function module 3D printing and adapted to the special features of the SEAMHex. This allows an optimal net planning depending on the component, machine and process model. The planning of 5-axis printing processes is possible to produce complex geometries without support contours like a demonstrator structure in Figure 11. In addition, the model of a base component can be inserted, on which the print structure is to be applied. In the case of the above-mentioned demonstrator structure, a curved base structure was inserted in the software to print on the demonstrator structure.
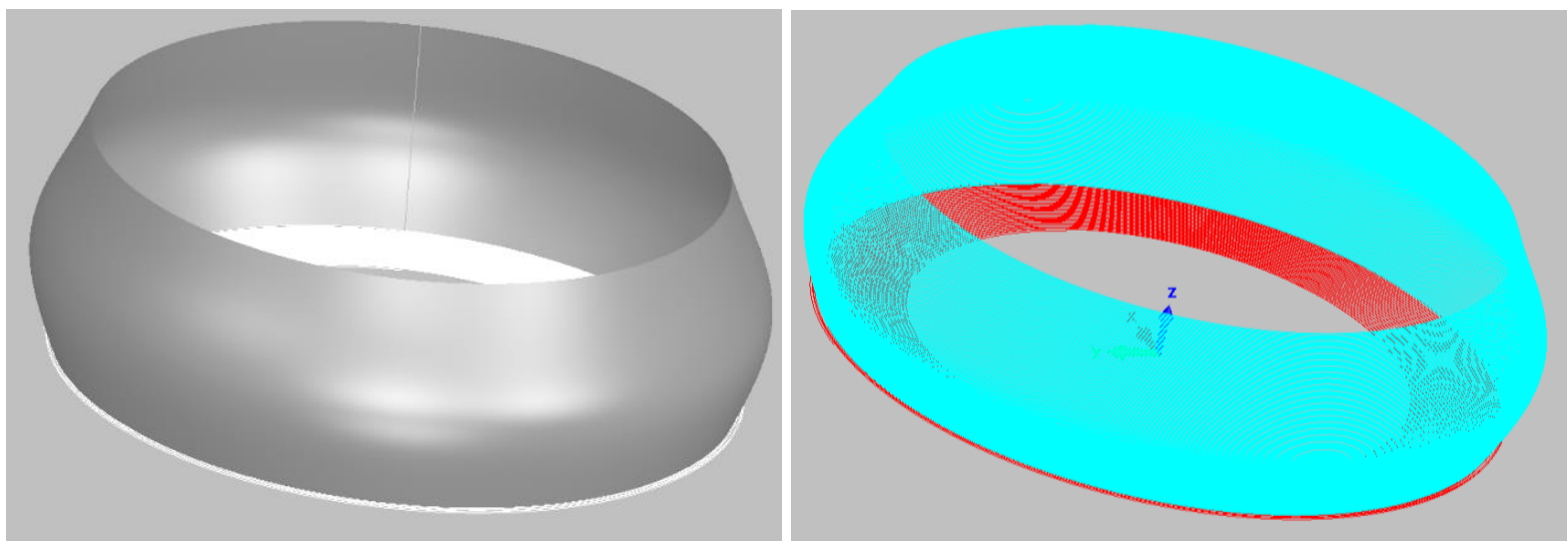

Figure 11: Demonstrator component (left) and sliced component in DCAM (right) 


\section{Positioning Accuracy and Printing Quality}

High printing quality requires high positioning accuracy of the hexapod and the $Z$-axis. To achieve the required accuracy of $\pm 100 \mu \mathrm{m}$ of the SEAMHex, the parameters of the kinematic transformation in the CNC must be adjusted, as deviations to the CAD model parameters occur after assembly. To evaluate the positioning accuracy of the 7-axis motion unit, two approaches were applied: the direct measurement along a continuous spatial path using the Double-Ball-Bar (DBB) measuring device as well as the indirect assessment by printing a test workpiece.

The DBB is a length-measuring device with a resolution of $\pm 0.1 \mu \mathrm{m}$ that is installed with the help of two magnetic spherical joints between tool centre point (TCP) and printing bed, Figure 12 left. For that, a plate of steel is mounted to the spindle nose and a magnetic three-point fixture attached to the platform, Figure 12 right. In the controller, two local coordinate systems for the DBB measurement are defined; one locating at the nominal position of the middle point of the three-point fixture and one at the nominal position of the middle ball of the measuring paths, which is about $60 \mathrm{~mm}$ underneath the steel plate.
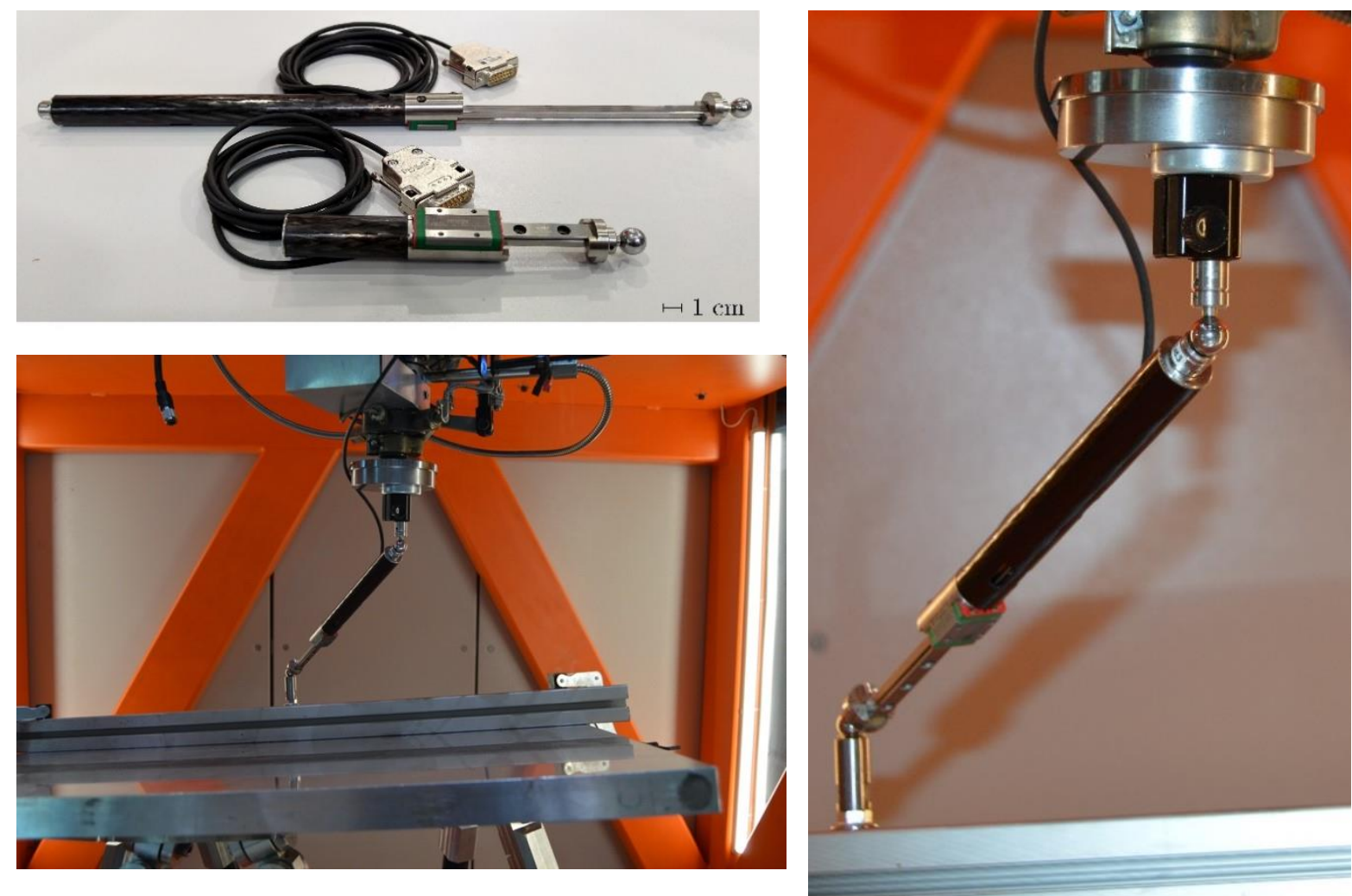

Figure 12: Double-Ball-Bars in different lengths, DBB assembled at SEAMHex

For the measurement, 3 spherical paths are generated and optimised with a nominal radius of $300 \mathrm{~mm}$ for the spatial DBB measurement by applying the path generation approach, as described in [21, 22]. The paths are visualized in Figure 13; in order from left to right, they are denoted by $p_{1}, p_{2}, p_{3}$. During the measurement, the DBB measuring data is continuously acquired within the control system. Hereby, axis lengths are captured synchronously. 

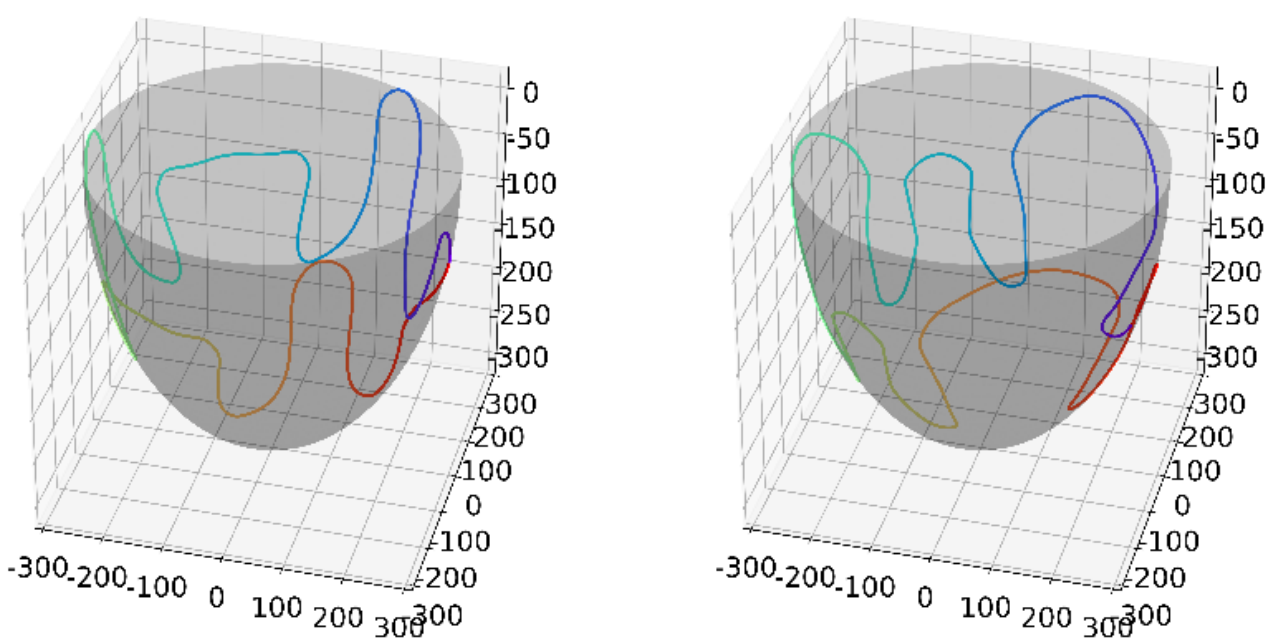

Figure 13: Spherical paths for continuous DBB measurement on SEAMHex (unit: $\mathrm{mm}$ )

The first DBB measurements shows large positioning deviations, Figure 14 top. To reduce these deviations, the relevant kinematic parameters were measured at the machine by different methods, first manually using touch probes and, in addition, with the photogrammetric approach [23]. The manufacturing tolerances of the welding frame led to deviations especially in the area of the shoulder joint coordinates on the hexapod. A DBB measurement after applying corrected kinematic parameters to the machine shows a significant improvement of the movement accuracy. As Figure 14 presents, the accuracy before the parameter corrections lies in range of millimetres and it is improved by factor 10 after calibration. Now, a positioning accuracy in range of $\pm 100 \mu \mathrm{m}$ is currently achieved on SEAMHex. Hereby, the measurement upon a continuous spatial path with the help of the extended DBB allows a fast assessment of the movement accuracy and the improvement after parameter change. The method can also be used to ensure movement accuracy in production breaks, after crash or after changes to the machine setup.
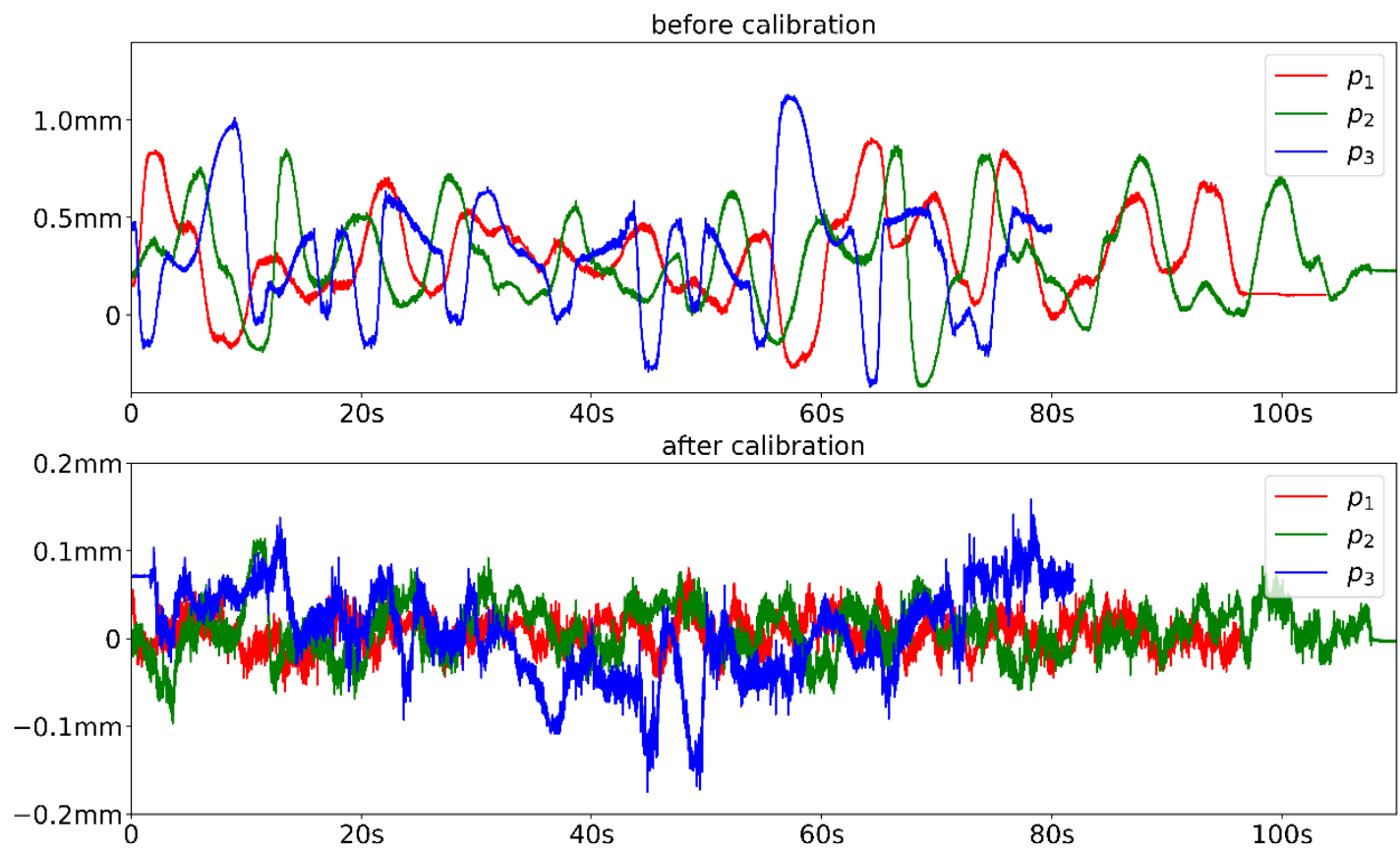

Figure 14: Positioning error for different measurement paths of the DBB before (top) and after (bottom) the machine calibration (different scale)

A second approach applied to the machine is the indirect accuracy assessment by producing a test workpiece. By checking defined geometric properties of a printed test workpiece with vernier calipers, the accuracy of machine movement can be evaluated. Geometric properties can be component dimensions, bead widths and corner radii, for example. Furthermore, the process quality can be 
controlled by means of the bead adhesion in the workpiece. Dimensional accuracy is also strongly correlated by the temperature and material, some results to the used material in the workpiece below are summed up in another paper [8]. The first test workpiece printed on the SEAMHex is a reinforced rectangular ribbed carrier printed with 3 dof $(2.5 \mathrm{D})$. To check the accuracy of movement even with several degrees of freedom, this ribbed support can also be printed on a curved surface. The result is shown in Figure 15.

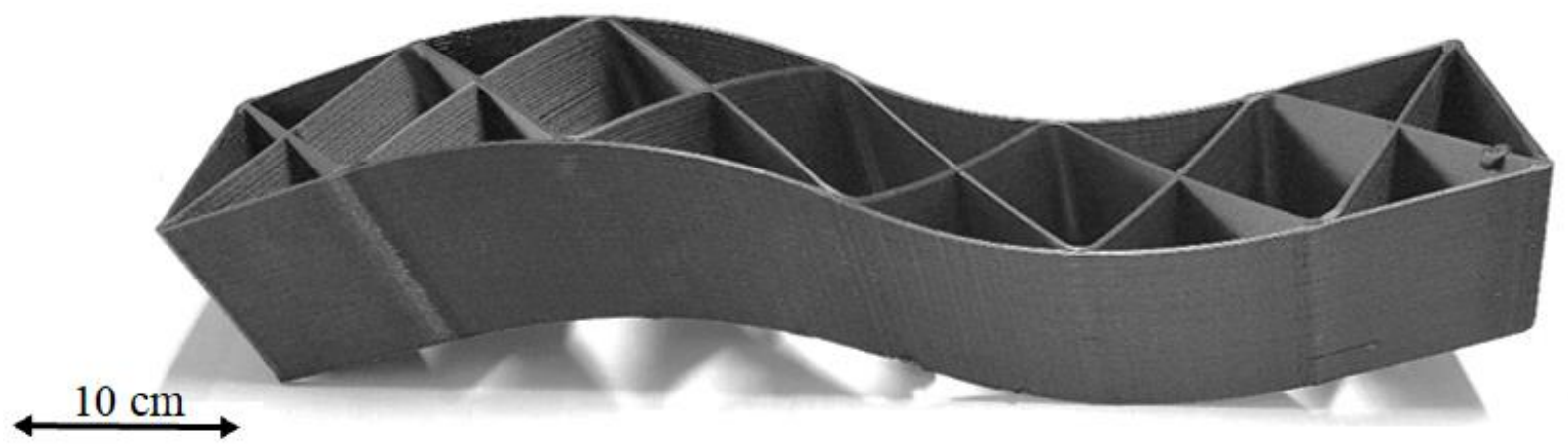

Figure 15: Demonstrator structure printed upon a curved based structure by using 4 of the 6 available degrees of freedom

\section{Conclusion}

A fast and accurate hexapod parallel kinematic SEAMHex has been developed that allows fast 6D additive manufacturing with moving workpiece and brings the benefits of the SEAM technology into application. Hereby, eccentric joints provide high accuracy and stiffness and the commercial numeric control allows G-code-based programming. Parts are sliced by an adapted CAM software and can be printed without support structures due to the 6-dof movement. The machine accuracy has been validated by the help of an extended double-ball-bar measuring device and by printing a test workpiece.

\section{Author Contributions}

Printing process and plasticizing unit, M. Kausch, L. Jankowsky; Hexapod design and control, C. Friedrich, T. Wiese; DBB measurement, C. Friedrich; Project Supervision S. Ihlenfeldt, W.-G. Drossel. All authors have read and agreed to the published version of the manuscript.

\section{Patents}

Fraunhofer Gesellschaft Forschung: John, C.; Sauerstein, T.; Kausch, M.; Blase, J. \& Uhlmann, S.: Regel- und Verschlusseinrichtung für extrudierten plastisch verformbaren Werkstoff. 21.09.2018 (DE102018216152A1)

\section{Acknowledgments}

Although the work was not directly funded, the authors are grateful to the German Research Foundation (DFG) who supported the following projects, which are basis for this work: "Measurement and Evaluation of the Spatial Positioning Accuracy in Multi-Axis Machine Tools in Operational Conditions" (No. 316667363), "Basics for the Usage of Eccentric Joints in Parallel Kinematic Machine Tools" (No. 53530748) as well as "Fundamentals for Structure-integrated Measurement and Control-integrated Processing of Spatial Forces and Torques in Machine Tools" (No. 202081830). In addition, the authors want to thank the TU Dresden Institute for Mechatronic Engineering and the Fraunhofer Institute for Machine Tools and Forming Technology for making this collaborative work possible. Finally, the authors want to thank the editors and reviewers for their helpful comments and constructive suggestions with regard to the revision of the paper. 


\section{References}

[1] Calignano, F., Manfredi, D., Ambrosio, E. P., Biamino, S., Lombardi, M., Atzeni, E., Salmi, A., Minetola, P., luliano, L., and Fino, P.: Overview on additive manufacturing technologies. Proceedings of the IEEE, 105 (4): 593-612, 2017.

[2] Dehghan, A., Namdari, N., Behrouz, M., and Behzad, F.: Additive Manufacturing Methods A Brief Overview. Journal of Scientific and Engineering Research, 5 (8): 123-131, 2018.

[3] Pham, D. T. and Gault, R. S.: A comparison of rapid prototyping technologies. International Journal of machine tools and manufacture, 38 (10-11): 1257-1287, 1998.

[4] Crump, S. S.: Fast, precise, safe prototypes with FDM. ASME, PED, 50: 53-60, 1991.

[5] Blase, J., John, C., M., K., and Witt, M.: Ultrafast 3D-Printing: Additive Manufacturing of Plastic Components in a High-Speed Process Using Standard Pellet Stock. Kunststoffe, pp. 8-10, 2019.

[6] Duty, C. E., Kunc, V., Compton, B., Post, B., Erdman, D., Smith, R., Lind, R., Lloyd, P., and Love, L.: Structure and mechanical behavior of Big Area Additive Manufacturing (BAAM) materials. Rapid Prototyping Journal, 2017.

[7] Widmann, B.: SEAM, ein ultraschnelles 3D-System. KunststoffExtra, pp. 12-13, 2019.

[8] Drossel, W., M., K., Blase, J., and Jankowsky, L.: Dimensional Accuracy of Extrusion-Based 3D Printing. In Proceedings of the 5th Fraunhofer Direct Digital Manufacturing Conference DDMC 2020. Fraunhofer-Verlag, Stuttgart, 2020.

[9] Merlet, J.-P.: Parallel robots, Vol. 74. Springer Science \& Business Media, 2012.

[10] Siciliano, B. and Khatib, O. editors. Handbook of robotics. Springer-Verlag Berlin Heidelberg, 2016.

[11] Neugebauer, R. editor. Parallelkinematische Maschinen: Entwurf, Konstruktion, Anwendung (VDI-Buch). Springer, 2006.

[12] Großmann, K., Kauschinger, B., and Szatmari, S.: Kinematic calibration of a hexapod of simple design. Production Engineering, 2 (3): 317-325, 2008. ISSN 0944-6524.

[13] Kauschinger, B.: Verbesserung der Bewegungsgenauigkeit an einer Parallelkinematik einfacher Bauart. PhD thesis, TU Dresden, 2006.

[14] Szatmari, S.: Kinematic Calibration of Parallel Kinematic Machines on the Example of the Hexapod of Simple Design. PhD thesis, TU Dresden, 2007.

[15] Großmann, K., Kauschinger, B., and Riedel, M.: Exzentrische Gelenke für parallelkinematische Werkzeugmaschinen. ZWF, 01-02: 25-32, 2012.

[16] Großmann, K. and Kauschinger, B.: Eccentric universal joints for parallel kinematic machine tools: variants and kinematic transformations. Production Engineering, 6 (4-5): 521-529, 2012. ISSN 0944-6524.

[17] Friedrich, C., Kauschinger, B., and Ihlenfeldt, S.: Stiffness evaluation of a hexapod machine tool with integrated force sensors. Journal of Machine Engineering, 20 (1): 58-69, 2020.

[18] Großmann, K., Kauschinger, B., and Riedel, M.: Optische Verlagerungsmessung an Kardangelenken. WT Online, 103: 402-409, 2013.

[19] Friedrich, C., Kauschinger, B., and Ihlenfeldt, S.: Spatial force measurement using a rigid hexapod-based end-effector with structure-integrated force sensors in a hexapod machine tool. Measurement, 145C: 350-360, 2019.

[20] Friedrich, C., Kauschinger, B., and Ihlenfeldt, S.: Decentralized structure-integrated spatial force measurement in machine tools. Mechatronics, 40: 17-27, 2016. 
[21] Kauschinger, B., Friedrich, C., Zhou, R., and Ihlenfeldt, S.: Fast Evaluation of the Volumetric Motion Accuracy of Multi-Axis Machine Tools using a Double-Ball-Bar. Journal of Machine Engineering, 20 (3): 44-62, 2020.

[22] Zhou, R., Kauschinger, B., and Ihlenfeldt, S.: Path generation and optimization for DBB measurement with continuous data capture. Measurement, 155: 107550, 2020.

[23] Riedel, M.: Methodik zur Modellierung von photogrammetrischen Messungen zur Charakterisierung der Genauigkeit von Werkzeugmaschinen. PhD thesis, TU Dresden, 2019. 